\title{
CRESCIMENTO DE Tectona grandis EM UM UMA PLANTAÇÃO NO MUNICÍPIO DE ALTA FLORESTA, MATO GROSSO
}

\author{
Fabricia Rodrigues da Silva ${ }^{1}$, Versides Sebastião de Moraes e Silva ${ }^{2}$, Soely Ovidio de Miranda ${ }^{3}$ \\ ${ }^{1}$ Eng $^{\mathrm{a}}$. Florestal, Mestranda UFMT, Cuiabá, MT, Brasil - engfabriciarodrigues@ gmail.com \\ ${ }^{2}$ Eng. Florestal, Dr., UFMT, MT, Brasil - versides@uol.com \\ ${ }^{3}$ Eng $^{\mathrm{a}}$ Florestal, M.Sc., UFMT, Cuiabá, MT, Brasil - soelymiranda @ yahoo.com.br \\ Recebido para publicação: 25/10/2012 - Aceito para publicação: 08/04/2014
}

\begin{abstract}
Resumo
Este trabalho teve como objetivo estudar o crescimento e incremento da espécie Tectona grandis L. f. mediante a análise do comportamento das variáveis diâmetro $(\mathrm{cm})$, altura $(\mathrm{m})$ e volume individual $\left(\mathrm{m}^{3}\right)$ ao longo do tempo, em uma plantação localizada em Alta Floresta, no estado de Mato Grosso. Os dados utilizados neste estudo são provenientes de uma plantação com amostras obtidas de 47 árvores dominantes, que serviram de base para a análise de tronco e a obtenção dos dados para a avaliação do crescimento no período. O crescimento médio para as variáveis diâmetro, altura e volume foi determinado pela aplicação de técnica de regressão para o ajuste e seleção da melhor equação que representasse o desenvolvimento padrão para as condições da plantação no local específico. Foram testadas equações para representar o crescimento médio da plantação, sendo que o modelo de Backman apresentou melhor desempenho para descrever a variável diâmetro. Para a variável altura, o modelo de Hoerl foi o selecionado, e para a variável volume o modelo de ChapmanRichards foi o escolhido. Os resultados são compatíveis quando comparados com outras regiões, o que permite concluir que o seu desenvolvimento é promissor e indica na direção da viabilidade da plantação dessa espécie.
\end{abstract}

Palavras-chave: Reflorestamento; análise de tronco; modelos de crescimento.

\begin{abstract}
Growth of Tectona grandis at a plantation located in Alta Floresta, Mato Grosso. This work aimed to study growth and increase of Tectona grandis L. f. species by analysis of behavior of the variables diameter, height and volume along time, in a plantation located in Alta Floresta, in the State of Mato Grosso. The data used in this study come from a plantation containing 47 dominant trees, which formed the basis for the analysis of trunks and the database for the evaluation of growth in the period. The average growth for the variables diameter, height and volume was determined applying the regression technique for adjustment and selection of the best equation to represent the standard development for the conditions of the plantation at the specific site. There were tested equations to represent the average growth of the plantation, being the Backman model the one that represented the best performance to describe the variable diameter. Hoerl model was selected for the variable height, and Chapman-Richards model was chosen for the variable volume. The results are compatible as compared to other regions, which allows to conclude that its development is promising and indicates the viability of planting the analyzed species.

Keywords: Reforestation; trunk analysis; growth models.
\end{abstract}

\section{INTRODUÇÃO}

O setor de florestas plantadas brasileiro apresenta um grande potencial de crescimento, por apresentar menor custo e ciclo de produção, maior produtividade e ativos menos sujeitos às oscilações do mercado financeiro, que são aspectos extremamente valorizados. Uma espécie que tem se destacado no setor florestal, principalmente no mercado externo, é a teca (Tectona grandis L. f.). No Brasil, ela é plantada em escala comercial nos estados do Mato Grosso, Amazonas, Acre e Pará (ABRAF, 2011). 
A teca, pertencente à família Lamiaceae, é uma árvore pioneira, decídua, de grande porte e copa circular. Sob condições de crescimento, desenvolve tronco retilíneo, com altura superior a $25 \mathrm{~m}$. Obtém melhor desempenho em locais com precipitação entre 1.270 e $2.540 \mathrm{~mm}$, com estação seca definida, temperatura mínima entre 13 e $17{ }^{\circ} \mathrm{C}$ e máxima entre 39 e $43{ }^{\circ} \mathrm{C}$. Desenvolve-se em diversos solos e formações geológicas, porém prefere solos drenados e profundos, com pH entre 6,5 e 7,5 (FAO, 1959; PANDEY; BROWN, 2000; KRISHNAPILLAY, 2000).

Entre as razões pelas quais a espécie é bastante utilizada em plantações estão o fato de ser de fácil propagação, estabelecimento e manejo e possuir madeira de excelente qualidade. É empregada em uma variedade de usos tradicionais e atuais, incluindo mobiliário, elementos estruturais, madeira, laminados e pisos. Sua alta resistência a produtos químicos é ideal para móveis de laboratório. Essas propriedades mantêm a teca como uma das principais espécies de madeira tropical de alto valor neste século (CAMINO; MORALES, 2013). Segundo Schuhli e Paludzyszyn Filho (2010), o interesse na espécie como uma alternativa aos plantios florestais vem crescendo muito, atualmente.

O estudo do crescimento possibilita conhecer o potencial de produção florestal de um determinado sítio e, com isso, orientar a tomada de decisões sob vários aspectos, para o atendimento da demanda do mercado (HOSOKAWA et al., 1998).

Prodan et al. (1997) aponta que o crescimento é o incremento gradual de um organismo, população ou objeto em um determinado período de tempo. É influenciado por suas características genéticas e sua inter-relação com o meio ambiente, como os fatores climáticos e do solo, as características topográficas, cuja soma representa a qualidade do local.

$\mathrm{O}$ crescimento em diâmetro, altura, área basal e volume de uma árvore apresenta comportamento semelhante ao longo do tempo. Em forma gráfica, parece uma curva sigmoidal, em que a primeira fase corresponde à idade juvenil, a segunda à idade madura e a terceira à idade senil (velha). Cada fase mantém um ritmo de crescimento característico da vida total da árvore e juntas formam a curva de crescimento (IMAÑA-ENCIMAS et al., 2005).

Alguns trabalhos sobre modelos de crescimento para teca foram desenvolvidos em Mato Grosso (SILVA, 2012; PELISSARI et al., 2013). No entanto, são muito reduzidos, tornando necessária uma melhor avaliação do desempenho dessa espécie. Com isso, o objetivo deste trabalho foi ajustar modelos de crescimento para as variáveis diâmetro $(\mathrm{cm})$, altura $(\mathrm{m})$ e volume individual $\left(\mathrm{m}^{3}\right)$ em função da idade, no município de Alta Floresta, Mato Grosso.

\section{MATERIAIS E MÉTODOS}

O estudo foi desenvolvido em um povoamento homogêneo de teca, com 1.365,16 ha implantados de 1996 a 2004, na Fazenda IDC, localizada no município de Alta Floresta, região norte do estado de Mato Grosso, entre as coordenadas $9^{\circ} 59^{\prime} 33.54^{\prime \prime}$ S 56 $50^{\circ} 58.48^{\prime \prime} \mathrm{W}, 10^{\circ} 05^{\prime} 45.96^{\prime \prime}$ S

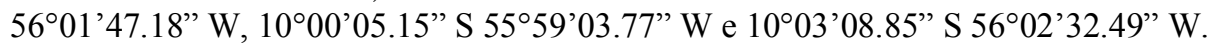

O clima da região é do tipo Awi, segundo a classificação de Köppen, ou seja, tropical chuvoso, alcançando elevado índice pluviométrico no verão, podendo atingir médias às vezes superiores a $2.750 \mathrm{~mm}$, e um inverno seco, predominando altas temperaturas. A temperatura média anual fica em torno de $26{ }^{\circ} \mathrm{C}$. Os solos que recobrem a região onde está localizada a propriedade foram descritos como Argissolos Vermelho-Amarelo distrófico (BRASIL, 1980; IBGE, 1997).

A propriedade está inserida na bacia do rio Teles Pires, que é o principal curso d'água da região.

As mudas originadas de sementes foram plantadas na forma de "stamp". Para o plantio, o solo foi descompactado, arado e nivelado, não havendo adubação no preparo do solo ou durante a condução do povoamento. A manutenção de capinas e roçadas foi constante durante os três primeiros anos, para eliminar a competição da teca com outras espécies. As desramas ocorreram a partir do segundo ano após o plantio e os desbastes, do tipo seletivo, foram executados aos seis e aos onze anos, com a remoção média de $25 \%$, do número de árvores por hectare.

Para a coleta dos dados, foram utilizadas como referência as 236 parcelas permanentes instaladas na área. Em cada uma das parcelas inventariadas, foi feita a análise estatística descritiva, o que tornou possível determinar o porte das árvores de altura dominante $\left(\mathrm{H}_{\mathrm{dom}}\right)$, utilizando o conceito de Naslund $\left(\mathrm{D}_{\mathrm{dom}} \geq \bar{D}+1,5 \mathrm{sd}\right)$. De posse dessas informações de referência, foi possível a identificação e localização 
de cada uma das árvores-amostra no campo. Foram selecionadas 47 árvores, cobrindo toda a variação aparente do local (sítio) e idade.

Das árvores-amostras, foram determinadas, mediante análise de tronco completa (anatro), as informações necessárias para a obtenção do crescimento. Na anatro, foi empregada a metodologia descrita por Barusso (1977).

Com os dados de diâmetro $(\mathrm{cm})$, altura $(\mathrm{m})$ e volume individual $\left(\mathrm{m}^{3}\right)$ por idade, tornou-se possível ajustar os modelos de crescimento para cada uma das variáveis, para representar o crescimento médio da plantação, cuja análise estatística descritiva está apresentada na tabela 1.

Tabela 1. Análise estatística descritiva do diâmetro a 1,30 m do solo (DAP) em centímetros e altura (H) em metros das árvores-amostra, de três a treze anos de idade, em Mato Grosso.

Table 1. Descriptive statistical analysis of the diameter at $1,30 \mathrm{~m}$ from the ground (DBH) in centimeters and height $(\mathrm{H})$ in meters, of trees from three to thirteen years old, in Mato Grosso.

\begin{tabular}{lcccccccccccc}
\hline \multirow{2}{*}{$\begin{array}{l}\text { Idade } \\
\text { (ano) }\end{array}$} & \multicolumn{2}{c}{ Mínimo } & \multicolumn{2}{c}{ Média } & \multicolumn{2}{c}{ Mediana } & \multicolumn{2}{c}{ Máximo } & \multicolumn{3}{c}{$\begin{array}{c}\text { Desvio } \\
\text { padrão }\end{array}$} & \multicolumn{2}{c}{$\begin{array}{c}\text { Coeficiente de } \\
\text { variação (\%) }\end{array}$} \\
\cline { 2 - 14 } & $\mathbf{D A P}$ & $\mathbf{H}$ & DAP & H & DAP & H & DAP & H & DAP & H & DAP & H \\
\hline 3 & 2,45 & 3,80 & 8,60 & 9,71 & 8,85 & 9,91 & 11,50 & 14,15 & 1,83 & 1,90 & 3,10 & 2,85 \\
4 & 5,85 & 7,20 & 10,79 & 11,93 & 10,70 & 11,90 & 13,95 & 16,00 & 1,70 & 1,75 & 2,30 & 2,14 \\
5 & 8,05 & 8,25 & 12,53 & 13,57 & 12,25 & 13,70 & 16,00 & 16,78 & 1,66 & 1,68 & 1,93 & 1,81 \\
6 & 9,85 & 9,20 & 13,87 & 14,59 & 13,70 & 14,45 & 17,40 & 18,35 & 1,69 & 1,78 & 1,78 & 1,78 \\
7 & 11,25 & 11,20 & 14,95 & 15,53 & 14,80 & 15,70 & 18,50 & 19,12 & 1,69 & 1,77 & 1,65 & 1,66 \\
8 & 12,65 & 13,20 & 15,88 & 16,22 & 15,80 & 16,10 & 19,00 & 19,10 & 1,61 & 1,43 & 1,62 & 1,41 \\
9 & 13,55 & 13,50 & 16,59 & 16,34 & 16,55 & 16,50 & 19,55 & 18,82 & 1,68 & 1,21 & 2,12 & 1,55 \\
10 & 14,60 & 13,90 & 17,23 & 17,01 & 16,68 & 17,13 & 20,15 & 19,10 & 1,70 & 1,27 & 2,46 & 1,86 \\
11 & 15,75 & 17,25 & 17,63 & 18,00 & 17,80 & 17,88 & 19,70 & 18,90 & 1,45 & 0,67 & 3,35 & 1,51 \\
12 & 16,30 & 17,35 & 18,24 & 18,44 & 18,75 & 18,50 & 20,50 & 19,25 & 1,67 & 0,82 & 4,10 & 2,00 \\
13 & 16,85 & 17,50 & 18,88 & 18,84 & 19,15 & 18,70 & 21,40 & 19,80 & 1,82 & 0,95 & 4,31 & 2,25 \\
\hline
\end{tabular}

Foram ajustados oito modelos de regressão disponíveis na literatura (Hess, 2006; Milani, 2010; Silva, 2012), os quais são apresentados na tabela 2 .

Tabela 2. Modelos ajustados para o crescimento das variáveis diâmetro, altura e volume.

Table 2. Models adjusted for the growth of the variables diameter, height and volume.

\begin{tabular}{lcc}
\hline Número & Modelo matemático & Autor \\
\hline 1 & $\ln (y)=\beta_{0}+\beta_{1} * \ln x_{i}+\beta_{2} * \ln ^{2} x_{i}$ & Backman \\
2 & $y=\beta_{0} *\left(1-\exp \left(-\beta_{1} * x_{i}\right)^{\beta_{2}}\right)$ & Chapman-Richards \\
3 & $\ln (y)=\beta_{0}+\beta_{1} * \ln x_{i}+\beta_{2} * x_{i}$ & Gram \\
4 & $\ln (y)=\beta_{0}+\beta_{1} *\left(1 \mid x_{i}\right)+\beta_{2} * \ln x_{i}$ & Hoerl \\
5 & $y=\beta_{0} *\left(1-\exp \left(-\beta_{1} * x_{i}\right)\right)$ & Mitscherlich \\
6 & $\ln (y)=\beta_{0}+\beta_{1} * x_{i}+\beta_{2} * x_{i}{ }^{2}+\beta_{3} * x_{i}^{3}$ & Moissev \\
7 & $\ln (y)=\beta_{0}+\beta_{1} *\left(x_{i}^{-1}\right)$ & Schumacher \\
8 & $\ln (y)=\beta_{0}+\beta_{1} *\left(x_{i}^{-1}\right)+\beta_{2} *\left(x_{i}^{-1}\right)^{2}+\beta_{3} *\left(x_{i}^{-1}\right)^{3}$ & Spillman \\
\hline
\end{tabular}

Ln: logaritmo natural; y: variável dependente (DAP, H e V); x: variável independente (idade); $\beta_{0}, \beta_{1}$, $\beta_{2}$ e $\beta_{3}$ : coeficientes a serem estimados.

Para os ajustes dos modelos matemáticos selecionados, utilizaram-se as rotinas do pacote estatístico Statistical Analysis System (SAS), com o Método dos Quadrados Mínimos para os modelos lineares e o algoritmo de Marquardt para os modelos não lineares.

Para selecionar o melhor modelo, observaram-se os critérios estatísticos definidos pelo coeficiente de determinação ajustado $\left(\mathrm{R}^{2}{ }_{\mathrm{aj}}\right)$, erro padrão da estimativa $\left(\mathrm{S}_{\mathrm{yx}} \%\right)$ e valor de $\mathrm{F}$. Também foi analisada a significância dos coeficientes de regressão $(\beta)$ e a análise gráfica dos resíduos. Para corrigir o erro sistemático (ou discrepância logarítmica) na estimativa da variável dependente, causado pela linearização do modelo logarítmico, foi aplicado o fator de correção de Meyer (Fm), utilizando a seguinte fórmula: 


$$
F m=e^{0,5 * Q M_{\text {res }}}
$$

em que: $\mathrm{QM}_{\text {res. }}=$ quadrado médio dos resíduos;

e = base do logaritmo natural $(2,718281829 \ldots)$.

O incremento corrente foi determinado pela primeira derivada da função de crescimento e o incremento médio pela razão do crescimento pela idade.

\section{RESULTADOS E DISCUSSÃO}

\section{Crescimento do diâmetro}

Para a análise do crescimento do diâmetro, foram testados os modelos descritos na tabela 2. Os resultados dos ajustes dos modelos para o crescimento em diâmetro são apresentados na tabela 3.

Tabela 3. Os estimadores dos parâmetros, ajuste $\left(\mathrm{R}^{2}{ }_{\mathrm{aj}}\right)$, erro padrão da estimativa $\left(\mathrm{S}_{\mathrm{yx}} \%\right)$ e estatística $\mathrm{F}$, para cada equação.

Table 3. Parameter estimators, adjust $\left(\mathrm{R}^{2}{ }_{\mathrm{aj}}\right)$, estimative standard error $\left(\mathrm{S}_{\mathrm{yx}} \%\right)$, $\mathrm{F}$ statistic, for each equation.

\begin{tabular}{lccccccccc}
\hline Equação & Autor & $\mathbf{b}_{\mathbf{0}}$ & $\mathbf{b}_{\mathbf{1}}$ & $\mathbf{b}_{\mathbf{2}}$ & $\mathbf{b}_{\mathbf{3}}$ & $\mathbf{R}_{\mathbf{A j}}^{\mathbf{2}}$ & $\mathbf{S}_{\mathbf{y x}} \mathbf{\%}$ & $\mathbf{F}$ \\
\hline 1 & Backman & $-2,308^{*}$ & $1,771^{*}$ & $-0,145^{*}$ & - & 0,877 & 12,572 & $923,565^{*}$ \\
2 & Chapman- & $19,984^{*}$ & $0,017^{*}$ & $1,025^{*}$ & - & 0,861 & 12,521 & $804,565^{*}$ \\
3 & Richards & $-1,163^{*}$ & $0,989^{*}$ & $-0,006^{*}$ & - & 0,859 & 12,589 & $794,583^{*}$ \\
4 & Gram & $0,985^{*}$ & $-9,764^{*}$ & $0,410^{*}$ & - & 0,855 & 12,770 & $768,513^{*}$ \\
5 & Hoerl & $20,184^{*}$ & $0,016^{*}$ & - & - & 0,861 & 12,507 & $1209,548^{*}$ \\
6 & Mitscherlich & Moissev & $0,691^{*}$ & $0,057^{*}$ & $-0,001^{*}$ & $0,000002^{*}$ & 0,844 & 13,259 & $527,948^{*}$ \\
7 & Schumacher & $2,923^{*}$ & $-22,460^{*}$ & - & - & 0,807 & 14,744 & $815,954^{*}$ \\
8 & Spillman & $3,222^{*}$ & $-49,140^{*}$ & $464,879^{*}$ & $-1976,537^{\mathrm{ns}}$ & 0,860 & 12,542 & $601,330^{*}$ \\
\hline
\end{tabular}

ns: não significativo; *: significativo ao nível de $5 \%$ de probabilidade.

Os resultados mostram que, de forma geral, as equações apresentaram ajustes $\left(R^{2} a j\right)$ com valores acima de 0,80 . Os modelos também proporcionaram valores semelhantes para o erro padrão da estimativa, o que indica pouca diferença no ajuste dos modelos.

Quanto à estatística F, esse parâmetro se mostrou bastante elevado em todas as equações significativo ao nível de 5\% de probabilidade. Assim, como os modelos de Backman e Mitscherlich apresentaram maior coeficiente de determinação e menor erro padrão da estimativa, procedeu-se a análise gráfica dos resíduos.

Na distribuição gráfica dos resíduos entre o diâmetro observado em cada idade e o estimado pelo modelo, pode-se verificar que o modelo de Backman se destaca, devido à distribuição homogênea dos resíduos, como mostra a figura 1.

A análise da tabela 3 e da figura 1 mostra o modelo de Backman como o mais adequado para descrever o crescimento do diâmetro, por apresentar uma superioridade no coeficiente de determinação ajustado $(0,877)$, menor erro padrão $(12,572 \%)$ e uma melhor distribuição dos resíduos.

Estimou-se o crescimento do diâmetro no período de 1998 a 2011 (13 anos), cujo padrão é mostrado na figura 1, na qual se observa que este é mais acelerado nos cinco anos iniciais da série, seguido por mudança na tendência do crescimento. No entanto, não houve ponto de culminância do crescimento em diâmetro, indicando que as árvores não alcançaram a fase de assíntota, ou seja, aos treze anos está em franco desenvolvimento. Isso se deve às características da espécie, por ser de ciclo longo, e também ao fato de a qualidade do sítio ser favorável ao desenvolvimento da espécie.

\section{Crescimento em altura}

$\mathrm{Na}$ análise do crescimento da altura, foram testados os modelos matemáticos relacionados na tabela 2. Os resultados dos ajustes dos modelos para o crescimento em altura são apresentados na tabela 4.

Os resultados da tabela 4 mostram que, de maneira geral, os modelos apresentaram ajuste $\left(R^{2} a j\right)$ e erro padrão da estimativa em torno de 0,85 e $12 \%$, respectivamente, assim como a estatística $\mathrm{F}$ se 
mostrou bastante elevada para todos. Como os modelos de Hoerl e Mitscherlich obtiveram maior coeficiente de determinação e menor erro padrão da estimativa, procedeu-se à análise gráfica dos resíduos.
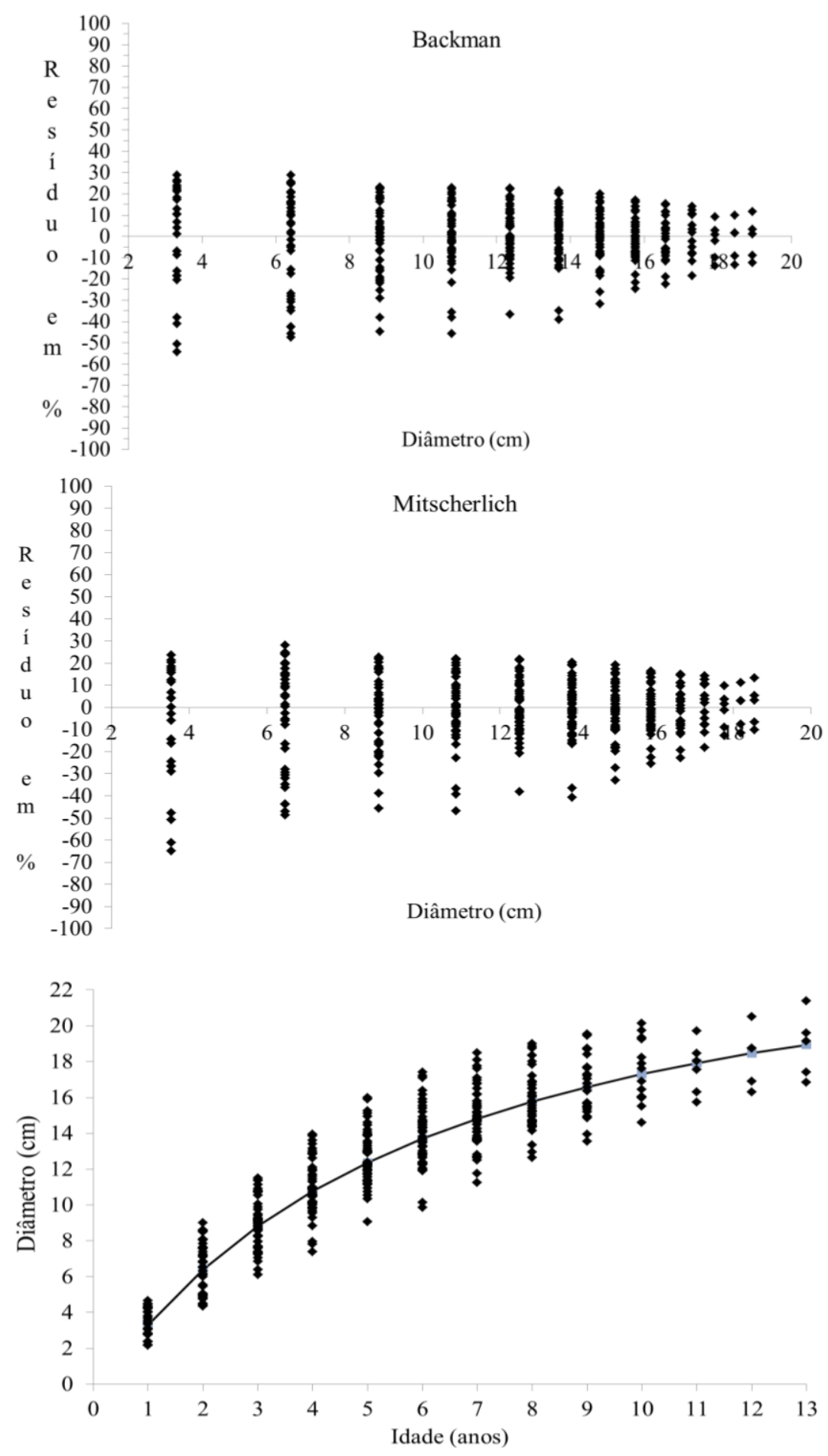

Figura 1. Distribuição dos resíduos e crescimento do diâmetro.

Figure 1. Distribution of the residues and diameter growth. 
Tabela 4. Estimadores dos parâmetros: ajuste $\left(\mathrm{R}_{\mathrm{aj}}^{2}\right)$, erro padrão da estimativa $\left(\mathrm{S}_{\mathrm{yx}} \%\right)$ e estatística $\mathrm{F}$, para cada equação.

Table 4. Parameter estimators: adjust $\left(\mathrm{R}_{\mathrm{aj}}^{2}\right)$, estimative standard error $\left(\mathrm{S}_{\mathrm{yx}} \%\right)$ and $\mathrm{F}$ statistic, for each equation.

\begin{tabular}{|c|c|c|c|c|c|c|c|c|}
\hline Equação & Autor & $\mathbf{b}_{0}$ & $b_{1}$ & $\mathbf{b}_{2}$ & $\mathbf{b}_{3}$ & $\mathbf{R}^{2}{ }_{\mathrm{Aj}}$ & $\mathrm{S}_{\mathrm{yx}} \%$ & $\mathbf{F}$ \\
\hline 1 & Backman & $-3,101^{*}$ & $2,306^{*}$ & $-0,223^{*}$ & - & 0,864 & 11,683 & $815,775^{*}$ \\
\hline 2 & $\begin{array}{l}\text { Chapman- } \\
\text { Richards }\end{array}$ & $18,315^{*}$ & $0,024^{*}$ & $1,081^{*}$ & - & 0,865 & 11,654 & $820,424^{*}$ \\
\hline 3 & Gram & $-1,261^{*}$ & $1,075^{*}$ & $-0,009^{*}$ & - & 0,854 & 12,138 & $746,422^{*}$ \\
\hline 4 & Hoerl & $2,093^{*}$ & $-15,977^{*}$ & $0,187^{*}$ & - & 0,866 & 11,615 & $826,850^{*}$ \\
\hline 5 & Mitscherlich & $18,654^{*}$ & $0,022^{*}$ & - & - & 0,865 & 11,654 & $1230,205^{*}$ \\
\hline 6 & Moissev & $0,719^{*}$ & $0,062^{*}$ & $-0,001^{*}$ & $0,000002^{*}$ & 0,835 & 12,895 & $485,359^{*}$ \\
\hline 7 & Schumacher & $2,976^{*}$ & $-21,799^{*}$ & - & - & 0,855 & 12,084 & $1130,726^{*}$ \\
\hline 8 & Spillman & $3,148^{*}$ & $-38,796^{*}$ & $372,263^{*}$ & $-2196,860^{\text {ns }}$ & 0,866 & 11,604 & $621,736^{*}$ \\
\hline
\end{tabular}

ns: não significante; *: significativo ao nível de $5 \%$ de probabilidade.

Na figura 2, que representa a distribuição gráfica dos resíduos entre a altura observada em cada idade e a estimada pelo modelo, a equação de Hoerl se destaca, por apresentar menor tendenciosidade para superestimar os dados.

A análise da tabela 4 e da figura 2 mostra a equação de Hoerl como a mais adequada para descrever o crescimento em altura, pois apresenta superioridade no $\mathrm{R}_{\text {aj }}^{2}(0,866)$, menor erro padrão $(11,615 \%)$ e dispersão homogênea dos resíduos. Selecionado o modelo, estimou-se o crescimento em altura em função da idade, cujo resultado é mostrado na figura 2.

Observa-se que o crescimento em altura apresenta padrão sigmoidal, com crescimento inicial rápido até os 3 anos de idade, diminuindo gradativamente após isso. Nota-se, ainda, que a altura da teca neste estudo aumentou progressivamente com a idade.

\section{Crescimento do volume}

$\mathrm{Na}$ análise do crescimento do volume, foram testados os modelos matemáticos relacionados na tabela 2. Os resultados dos ajustes dos modelos para o crescimento em volume são apresentados na tabela 5 .

Tabela 5. Estimadores dos parâmetros: ajuste $\left(\mathrm{R}_{\mathrm{aj}}^{2}\right)$, erro padrão da estimativa $\left(\mathrm{S}_{\mathrm{yx}} \%\right)$ e estatística $\mathrm{F}$, para cada equação.

Table 5. Parameter estimators: adjust $\left(\mathrm{R}_{\mathrm{aj}}^{2}\right)$, estimative standard error $\left(\mathrm{S}_{\mathrm{yx}} \%\right)$ and $\mathrm{F}$ statistic, for each equation.

\begin{tabular}{|c|c|c|c|c|c|c|c|c|}
\hline Equação & Autor & $\mathbf{b}_{0}$ & $b_{1}$ & $\mathbf{b}_{2}$ & $\mathbf{b}_{3}$ & $\mathbf{R}_{\mathrm{Aj}}^{2}$ & $\mathrm{~S}_{\mathrm{yx}} \%$ & $\mathbf{F}$ \\
\hline 1 & Backman & $-14,342^{*}$ & $4,401^{*}$ & $-0,370^{*}$ & - & 0,826 & 22,622 & $482,947^{*}$ \\
\hline 2 & $\begin{array}{l}\text { Chapman- } \\
\text { Richards }\end{array}$ & $0,281^{*}$ & $0,014^{*}$ & $2,130^{*}$ & - & 0,826 & 22,628 & $482,645^{*}$ \\
\hline 3 & Gram & $-10,527^{*}$ & $2,122^{*}$ & $-0,011^{*}$ & - & 0,825 & 22,739 & $476,922^{*}$ \\
\hline 4 & Hoerl & $-4,548^{*}$ & $-39,477^{*}$ & $0,656^{*}$ & - & 0,825 & 22,727 & $477,540^{*}$ \\
\hline 5 & Mitscherlich & $53,202^{\mathrm{ns}}$ & $0,00003^{\text {ns }}$ & - & - & 0,806 & 23,938 & $630,277^{*}$ \\
\hline 6 & Moissev & $-5,864^{*}$ & $0,094^{*}$ & $-0,001^{*}$ & $0,000002^{*}$ & 0,824 & 22,744 & $357,768^{*}$ \\
\hline 7 & Schumacher & $-1,237^{*}$ & $-72,357^{*}$ & - & - & 0,806 & 23,895 & $633,100^{*}$ \\
\hline 8 & Spillman & $-0,741^{*}$ & $-135,330^{*}$ & $1993,906^{\mathrm{ns}}$ & $-16203,0^{\text {ns }}$ & 0,826 & 22,661 & $360,961^{*}$ \\
\hline
\end{tabular}

Os resultados mostram que, de forma geral, as equações apresentaram certa uniformidade para expressar o crescimento volumétrico da espécie estudada.

De acordo com a tabela 5, destacam-se os seguintes modelos: o de Chapman-Richards, que apresentou maior coeficiente de determinação ajustado $(0,826)$ e menor erro padrão $(22,628 \%)$, e o modelo de Backman, com valor de coeficiente de determinação ajustado de 0,826 e erro padrão de $22,622 \%$. Assim, para estes, procedeu-se à análise gráfica dos resíduos. 

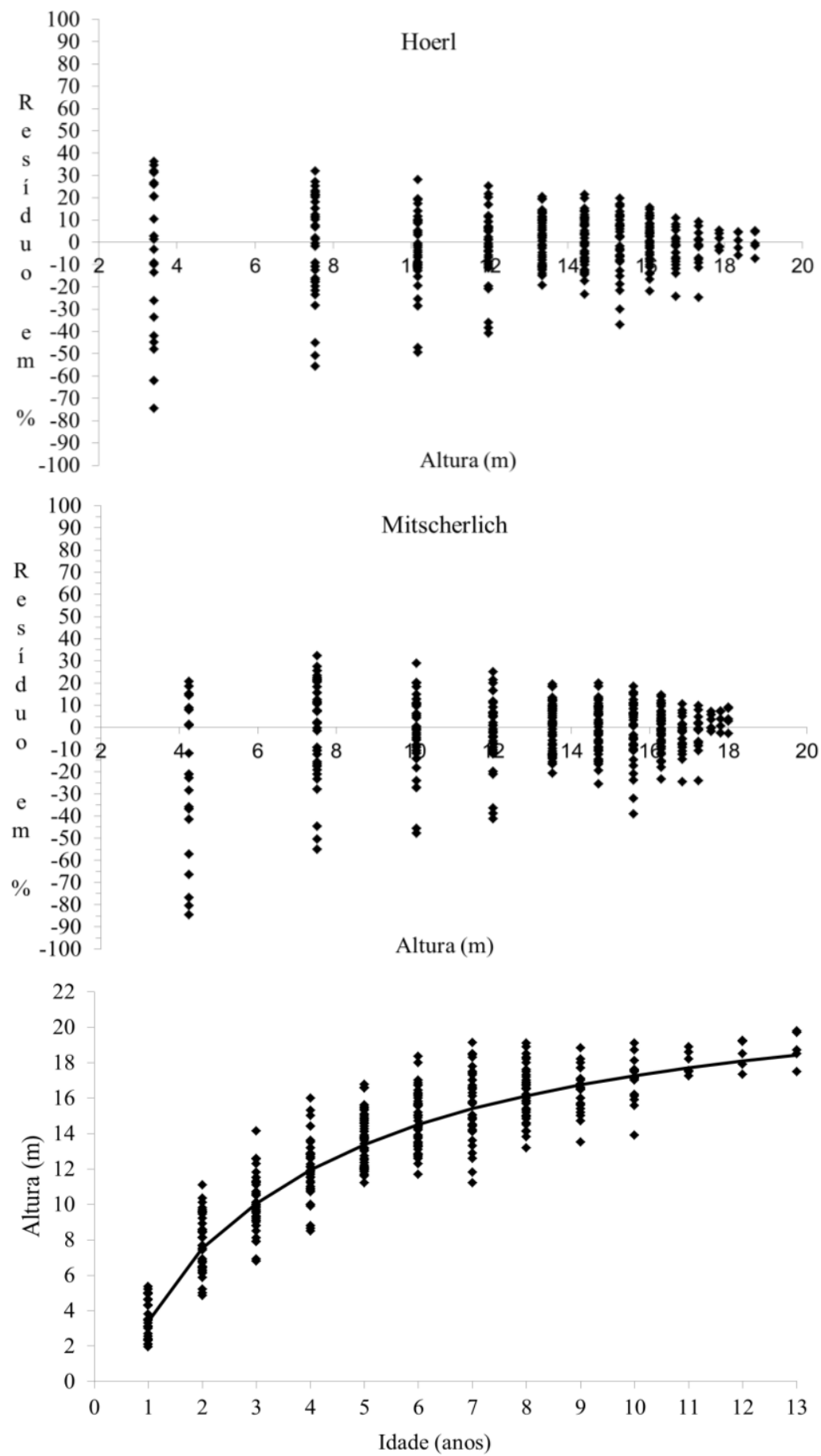

Figura 2. Distribuição dos resíduos e crescimento da altura.

Figure 2. Distribution of the residues and height growth.

Na figura 3, que representa a distribuição gráfica dos resíduos entre o volume observado em cada idade e o estimado pelo modelo, a equação de Chapman-Richards se destaca, devido à dispersão homogênea dos resíduos.

A tabela 5 e a figura 3 mostram o modelo de Chapman-Richards como o mais adequado para descrever o crescimento do volume, por apresentar uma superioridade no $R^{2}{ }_{\text {aj }}(0,826)$, erro padrão com o 
menor valor $(22,628 \%)$ e dispersão com um padrão homogêneo dos resíduos. Em função disso, o modelo de Chapman-Richards foi o selecionado. Resultado semelhante foi obtido por Tonini et al. (2009), estudando o crescimento em volume, no município de Iracema, RR.
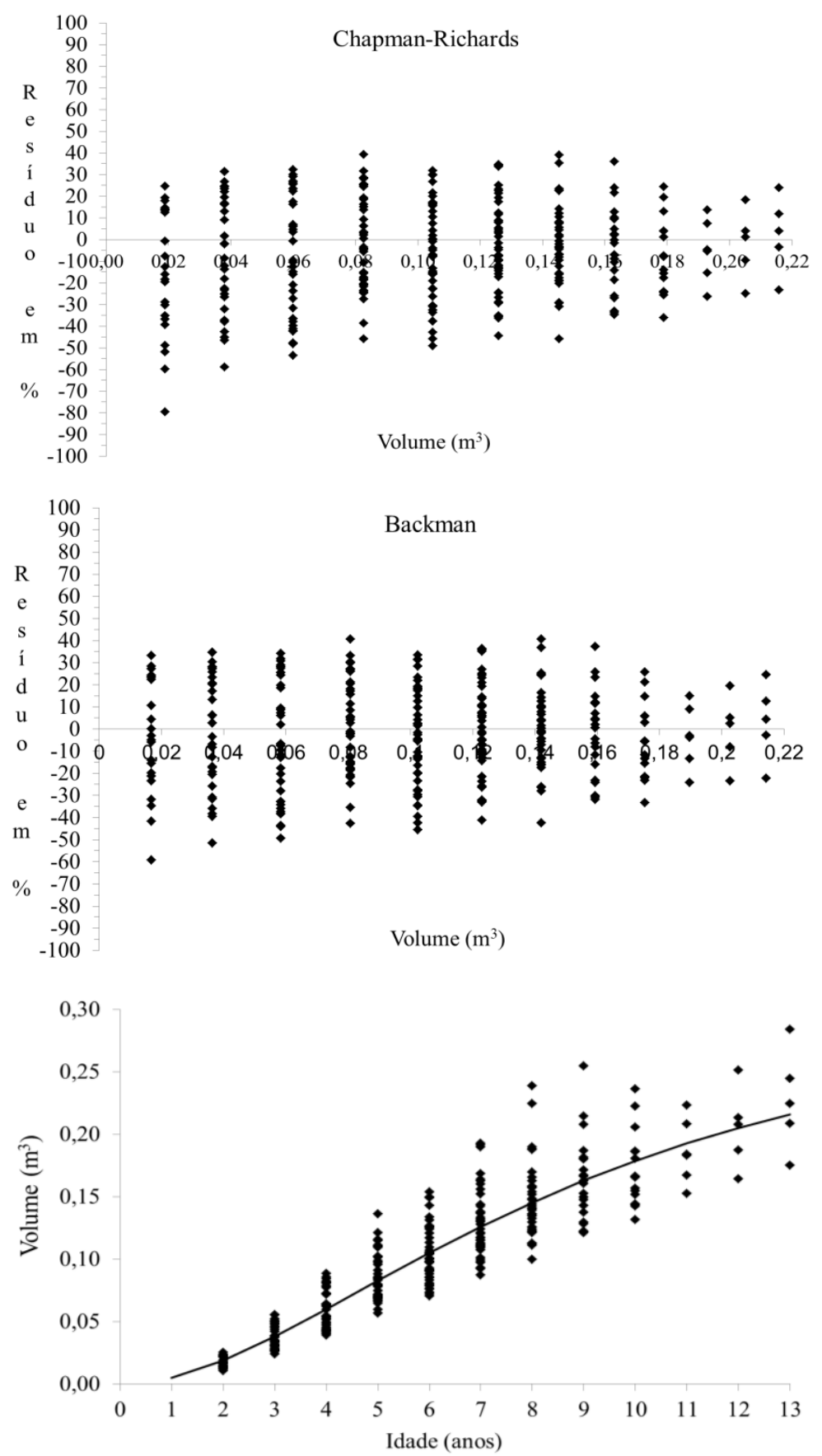

Figura 3. Distribuição gráfica dos resíduos e crescimento do volume.

Figure 3. Graphic distribution of the residues and volume growth.

Com o modelo ajustado, estimou-se o crescimento em volume em função da idade, cujo resultado é mostrado na figura 3. Observa-se que o padrão apresentado coincide com o padrão típico de 
crescimento das árvores, que é sigmoidal. O crescimento aumenta lentamente nos anos iniciais, porém com aumento exponencial ao longo dos 13 anos.

Para completar a análise do crescimento, foram calculados o incremento médio anual (IMA) e o incremento corrente anual (ICA) em diâmetro $(\mathrm{cm})$, altura $(\mathrm{m})$ e volume individual $\left(\mathrm{m}^{3}\right)$, cujos resultados são apresentados na tabela 6 .

Tabela 6. Incremento corrente anual (ICA) e incremento médio anual (IMA) para diâmetro (DAP), altura $(\mathrm{H})$ e volume $(\mathrm{V})$, por árvore, para Tectona grandis em Alta Floresta, Mato Grosso.

Table 6. Current annual increment (CAI) and average annual increase (AAI) for diameter (DBH), height (H) and volume (V), by each tree, for Tectona grandis in Alta Floresta, Mato Grosso.

\begin{tabular}{lccccccccc}
\hline Idade & $\begin{array}{c}\text { DAP } \\
(\mathbf{c m})\end{array}$ & $\begin{array}{c}\text { ICA DAP } \\
(\mathbf{c m})\end{array}$ & $\begin{array}{c}\text { IMA DAP } \\
(\mathbf{c m})\end{array}$ & $\begin{array}{c}\mathbf{H} \\
(\mathbf{m})\end{array}$ & $\begin{array}{c}\text { ICA H } \\
(\mathbf{m})\end{array}$ & $\begin{array}{c}\text { IMA H } \\
(\mathbf{m})\end{array}$ & $\begin{array}{c}\mathbf{V} \\
\left(\mathbf{m}^{\mathbf{3}}\right)\end{array}$ & $\begin{array}{c}\text { ICA V } \\
\left(\mathbf{m}^{\mathbf{3}}\right)\end{array}$ & $\begin{array}{c}\text { IMA V } \\
\left(\mathbf{m}^{\mathbf{3}}\right)\end{array}$ \\
\hline 1 & 3,3156 & 3,4852 & 3,3156 & 3,42 & 6,41 & 3,42 & 0,0051 & 0,0100 & 0,0051 \\
2 & 6,4085 & 2,7246 & 3,2043 & 7,53 & 3,01 & 3,76 & 0,0189 & 0,0170 & 0,0095 \\
3 & 8,8337 & 2,1577 & 2,9446 & 10,08 & 2,16 & 3,36 & 0,0381 & 0,0209 & 0,0127 \\
4 & 10,7767 & 1,7496 & 2,6942 & 11,95 & 1,63 & 2,99 & 0,0600 & 0,0225 & 0,0150 \\
5 & 12,3677 & 1,4464 & 2,4735 & 13,39 & 1,26 & 2,68 & 0,0826 & 0,0226 & 0,0165 \\
6 & 13,6930 & 1,2139 & 2,2822 & 14,51 & 1,00 & 2,42 & 0,1049 & 0,0217 & 0,0175 \\
7 & 14,8120 & 1,0310 & 2,1160 & 15,41 & 0,81 & 2,20 & 0,1259 & 0,0203 & 0,0180 \\
8 & 15,7669 & 0,8840 & 1,9709 & 16,14 & 0,67 & 2,02 & 0,1454 & 0,0186 & 0,0182 \\
9 & 16,5889 & 0,7638 & 1,8432 & 16,75 & 0,56 & 1,86 & 0,1630 & 0,0167 & 0,0181 \\
10 & 17,3014 & 0,6642 & 1,7301 & 17,27 & 0,48 & 1,73 & 0,1788 & 0,0149 & 0,0179 \\
11 & 17,9225 & 0,5805 & 1,6293 & 17,71 & 0,41 & 1,61 & 0,1928 & 0,0131 & 0,0175 \\
12 & 18,4665 & 0,5094 & 1,5389 & 18,09 & 0,36 & 1,51 & 0,2051 & 0,0115 & 0,0171 \\
13 & 18,9448 & 0,4486 & 1,4573 & 18,42 & 0,31 & 1,42 & 0,2159 & 0,0100 & 0,0166 \\
\hline
\end{tabular}

O crescimento em diâmetro obtido na plantação estudada aos 10 anos de idade $(17,3 \mathrm{~cm})$ foi superior ao obtido por Gyi e Tint (1998), que encontraram $12 \mathrm{~cm}$ na Índia e $6 \mathrm{~cm}$ na Tailândia, também aos 10 anos de idade. Chaves e Fonseca (1991) obtiveram crescimento em diâmetro de 11,3 a 12,5 cm na idade de 10 anos, para a América Central, resultado inferior ao encontrado neste estudo.

Tonini et al. (2009) obtiveram incremento médio anual de $1,27 \mathrm{~cm} /$ ano aos 7 anos para o município de Iracema (RR), resultado esse que difere do encontrado neste trabalho, em que o IMA foi de 2,11 (cm/ano), o que mostra que a espécie teve melhor crescimento nessa região da Amazônia, norte do estado de Mato Grosso.

Para plantações da espécie na Malásia em espaçamento de 3 x 4 m, Krishnapillay (2000) obteve crescimento de 4,0 $\mathrm{m}$ em altura no primeiro ano de idade, resultado este superior ao encontrado neste estudo, que foi de 3,42 m nessa mesma idade. Por outro lado, valores inferiores aos obtidos neste estudo foram observados nos trabalhos de Noor (2003) e Rossi et al. (2011). Para a idade de 2 anos, Noor (2003) obteve IMA de $0,72 \mathrm{~m} / \mathrm{ano}$ para a teca em Perlis, Malásia, enquanto que Rossi et al. (2011) obtiveram o valor de 1,03 m/ano aos 13 anos (Monte Dourado, PA). Neste estudo, o valor do IMA foi de 3,76 m/ano aos 2 anos e de 1,42 m/ano aos 13 anos.

O crescimento em volume obtido na plantação estudada aos 10 anos de idade $\left(0,178 \mathrm{~m}^{3}\right)$ foi superior ao obtido por Gyi e Tint (1998), que encontraram $0,02 \mathrm{~m}^{3}$ na Índia, aos 10 anos de idade. Rossi et al. (2011), em Monte Dourado, PA, obteve crescimento de $0,204 \mathrm{~m}^{3}$ aos 10 anos de idade, resultado superior ao encontrado neste estudo.

O incremento médio em volume aos 10 anos foi superior ao observado por Gyi e Tint (1998) em plantações de teca na Índia, com valor de $0,0149 \mathrm{~m}^{3} /$ ano.

\section{CONCLUSÕES}

- As equações testadas para estimar o crescimento em diâmetro (cm), altura (m) e volume individual $\left(\mathrm{m}^{3}\right)$ em função da idade apresenta resultados satisfatórios, exceto o modelo de Spillman, que não proporciona ajuste aceitável. 
- Os resultados de crescimento no local estudado demonstram que a espécie tem incrementos significativos, com taxas superiores aos plantios da região de origem.

\section{REFERÊNCIAS}

ASSOCIAÇÃO BRASILEIRA DE PRODUTORES DE FLORESTAS PLANTADAS (ABRAF). Anuário estatístico da ABRAF 2011. Brasília. 2011. 130 p. Disponível em: http://www.abraflor.org.br/estatisticas.asp. Acesso em: 02/09/2012.

BARUSSO, A. P. A determinação de funções de crescimento mediante análise de tronco. $122 \mathrm{p}$. Dissertação (Mestrado em Ciências Florestais) - Setor de Ciências Agrárias, Universidade Federal do Paraná, Curitiba, 1977.

BRASIL. Departamento Nacional da Produção Mineral. Levantamento de recursos naturais. Folha SC. 21. Juruena; geologia, geomorfologia, pedologia, vegetação e uso do potencial da terra. Rio de Janeiro: Gráfica Alvorada Ltda., 1980.

CAMINO, R.; MORALES, J. P. Las plantaciones de teca em América Latina: mitos y realidades. Turrialba, CATIE - Informe Técnico no 397, 2013. 392 p.

CHAVES, E.; FONSECA, W. Teca (Tectona grandis L.F.) árbol de uso múltiple em América Central. Turrialba, CATIE - Informe Técnico n ${ }^{\circ}$ 179, 1991. 47 p.

FOOD AND AGRICULTURE ORGANIZATION OF THE UNITED NATIONS (FAO). Elección de especies arbóreas para plantación. Roma: FAO, 1959. 375 p.

GYI, K. K.; TINT, K. Management Status of Natural Teak Forests. In Proc. Second Regional Seminar on Teak: Teak for the Future, 1995, Yangon, Nyanmar. FAO Regional Office for Asia and the Pacific, Bangkok, Thailand, 1998. p. 27 - 48.

HESS, A. F. Inter-relações no crescimento de Araucaria angustifolia (Bertol.) Kuntze em diferentes locais do Rio Grande do Sul. 177 f. Tese (Doutorado em Engenharia Florestal) - Universidade Federal de Santa Maria, Santa Maria, 2006.

HOSOKAWA, R. T.; MOURA, J. B.; CUNHA, U. S. Introdução ao manejo e economia de florestas. Curitiba: Editora UFPR, 1998. 162 p.

INSTITUTO BRASILEIRO DE GEOGRAFIA E ESTATÍSTICA (IBGE). Recursos naturais e meio ambiente: uma visão do Brasil. Departamento de Recursos Naturais e Estudos Ambientais. 2. ed. Rio de Janeiro, RJ: IBGE, 1997.

IMAÑA-ENCINAS, J.; SILVA, G. F.; PINTO, J. R. R. Idade e crescimento das árvores. Comunicações técnicas florestais, Brasília, UFB, Departamento de Engenharia Florestal, v. 7, n. 1, 2005. 43 p.

KRISHNAPILLAY, B. Silviculture and management of teak plantations. Unasylva. Roma, v. 51, n. 201, p. 14 - 21, 2000.

MILANI, E. J. Crescimento de Podocarpus lambertii Klotzsch ex Endl. em duas regiões fitogeográficas no estado do Rio Grande do Sul. 155 f. Tese (Doutorado em Engenharia Florestal) Universidade Federal de Santa Maria, Santa Maria. 2010.

NOOR, H. Growth of teak (Tectona grandis) on lateritic soil at Mata Aver forest reserve, Perlis. Journal of Tropical Forest Science, 15 (1), p. 190 - 198, 2003.

PANDEY, D.; BROWN, C. Teak: a global overview. Unasylva, Roma, v. 51, n. 201, p. 3 - 13, 2000.

PELISSARI, A. L.; CALDEIRA, S. F.; DRESCHER, R. Desenvolvimento quantitativo e qualitativo de Tectona grandis L. f. em Mato Grosso. Floresta e Ambiente, v. 20, p. 371 - 383, 2013.

PRODAN, M.; PETERS, R.; COX, F.; REAL, P. Mensura forestal. San José, Costa Rica: GTZ-IICA, 1997. $586 \mathrm{p}$. 
ROSSI, A. S.; DRESCHER, R.; PELISSARI, A. L.; LANSSANOVA, L. R. Relação hipsométrica e crescimento de Tectona grandis L. f. no município de Monte Dourado, PA. Scientia Forestalis, Piracicaba, v. 39, p. 301 - 307, 2011.

SCHUHLI, G. S.; PALUDZYSZYN FILHO, E. O cenário da silvicultura de teca e perspectivas para o melhoramento genético. Pesquisa Florestal Brasileira, v. 30, n. 63, p. 217 - 230, 2010.

SILVA, F. R. Crescimento e produção de Tectona grandis L. f. em um plantio no município de Alta Floresta, Mato grosso. 94 p. Dissertação (Mestrado em Ciências Florestais e Ambientais) Universidade Federal de Mato Grosso, Cuiabá, Mato Grosso, 2012.

TONINI, H.; COSTA, M. C. G.; SCHWENGBER, L. A. M. Crescimento da teca (Tectona grandis) em reflorestamento na Amazônia setentrional. Pesquisa Florestal Brasileira, Colombo, n. 59, p. 05 - 14, 2009. 
FLORESTA, Curitiba, PR, v. 44, n. 4, p. 577 - 588, out. / dez. 2014. Silva, F. R. da; Silva, V. S. de M. e; Miranda, S. O. de. 